\section{Academic background of oral and maxillofacial specialty trainees in the United Kingdom}

\author{
T. W. M. Walker, ${ }^{* 1}$ A. S. Tahim, ${ }^{1}$ C. K. Lau, ${ }^{2}$ T. Nyunt ${ }^{3}$ and P. Magennis ${ }^{4}$ \\ VERIFIABLE CPD PAPER
}

IN BRIEF
presents the results of a survey used to
profile the academic background of oral
and maxillofacial specialty trainees.
Suggests that oral and maxillofacial
surgery is not immune to the crisis in
academic surgery.
Reports that determining the numbers
of trainees, stages of trainees and their
potential sub specialty interests is useful
for manpower planning within a specialty.

\begin{abstract}
Introduction The academic profile of a specialty can be measured in a number of ways. In the selection process for entry into higher training in oral \& maxillofacial surgery (OMFS) and for interface fellowships in surgery trainees are awarded points for papers published, presentations, teaching and learning, grants and higher degrees. General information about career development can provide trainers, and trainees, with information and guidance. Methods A web-based questionnaire was developed and distributed via electronic mailing lists to all OMFS specialist trainees. Basic demographic information was collected. Previous, current and future career plans were questioned, as was academic background in terms of publications, presentations, prizes and research grants as well as further degrees and examinations. Results One hundred and five OMFS specialty registrar trainees (StR) replied (76.6\% response rate). 83.3\% were male and the average age of all trainees was 37 years old. $74.7 \%$ obtained a training post on the first application. $62.6 \%$ of trainees were keen to practice in trauma surgery. $76.6 \%$ were keen to undertake a fellowship. 20.9\% were keen to be involved in academia (teaching) and 9.9\% in academia (research). 22.1\% of trainees had obtained grants. Conclusion Those involved in appointing to training programmes will now be able to see the level of competition. Future applicants to training programmes in oral and maxillofacial surgery in the United Kingdom are now aware of the level of competition. OMFS is not immune to the 'academic crisis' that exists in other surgical specialties, and the completion of higher degrees and entry in to academic careers should be encouraged and supported among trainees with an interest.
\end{abstract}

\section{INTRODUCTION}

The academic profile of trainees in oral and maxillofacial surgery (OMFS) is important within the UK. Application procedures for specialist training and fellowships (cleft, head and neck, aesthetics and craniofacial surgery), place emphasis on research and education in terms of papers published, presentations, grants, involvement in curriculum development and higher degrees. Information regarding the academic achievements of current trainees will be of benefit to both trainees and trainers in terms of career development and workforce planning, with particular reference to establishing

\footnotetext{
'Dental Student, King's College, Guy's Hospital, London, SE1 9RT, UK; ${ }^{2}$ Speciality Registrar in Oral \& Maxillofacial Surgery, Royal London Hospital, London, UK ${ }^{3}$ Senior House Officer in Oral \& Maxillofacial Surgery, St George's Hospital, London, UK; ${ }^{4}$ Consultant Oral \&t Maxillofacial Surgeon, Liverpool, UK

${ }^{*}$ Correspondence to: Tom W. M. Walker

Email: Tomwmw@hotmail.com; Tel: +44 7976778434

Refereed Paper

Accepted 9 January 2013

DOI: 10.1038/sj.bdj.2013.484

${ }^{\circledR}$ British Dental Journal 2013; 214: 519-523
}

potential academic training pathways and research centres.

The academic profile of trainees within other specialties has been investigated. A survey of radiology trainees ${ }^{1}$ found 50\% of trainees had completed further degrees in addition to MBBS and royal college examinations. Approximately 20\% had published in national journals as first author while $80 \%$ had presented at national conferences. A telephone survey of plastic surgery senior house officers (SHOs) ${ }^{2}$ in 2003 revealed over 35\% had other degrees. Fifty-seven percent had at least one publication. Fifteen percent expressed a desire to undertake further research. This included, in order of popularity, an MD, MSc followed by a PhD.

Given the requirement for dual qualification, opportunities to complete formal research degrees may be limited in OMFS. Trainees are often undertaking second undergraduate degrees while colleagues in other surgical specialties may be conducting formal research. Trainees may be reluctant to pursue research degrees or educational qualifications which may further increase the amount of time in nonclinical training.

This paper investigates current oral and maxillofacial specialist trainees, exploring their academic backgrounds and qualifications, along with evaluation of their intentions to conduct future research and pursue academic careers.

\section{METHODS}

A web-based questionnaire was developed, based on previous studies ${ }^{1,2}$ and distributed via electronic mailing lists to all specialist trainees in OMFS over a four-month period. There were a total of 137 specialist trainees who could have replied.

Demographic information was limited to age and gender. The information collected was grouped into data regarding current training in general and academic achievements and intentions. Current training data investigated the experience of the trainees before beginning specialist training, subspecialty interests and desire to undertake clinical fellowships as well 
as information relating to application to specialist training. Academic information included numbers of publications, presentations, time out for research, grants awarded and research prizes.

\section{RESULTS}

A total of 105 trainees in OMFS completed the survey. The response rate was $76.6 \%$.

\section{Demographic information}

$83.3 \%$ were male and the age range was 30 to 47 with a mean of 37 years old.

\section{Current training}

$91.7 \%$ of trainees were on a specialist training programme. $74.7 \%$ obtained a place on the specialist training programme after one round of application. 19.8\% after two rounds of application. 4.4\% after three rounds and 1.1\% after four rounds of application.

$28.1 \%$ of speciality registrars (StRs) were in StR3 year, with 18.9\% in StR4, 15.7\% in StR5, 9\% in StR6, 9\% in StR7, and 7.9\% in StR8. 12.4\% were in their furst fellowship year and $1.1 \%$ was in their second fellowship year (Fig. 1).

Trainees were asked to select which subspecialty areas in which they were keen to practice in. The most frequent response was facial trauma with 62.6\%. 54.9\% were keen to practice in oncological surgery, 52.7\% in facial deformity and 47.3\% in skin surgery. $45.1 \%$ desired to practice in facial aesthetics and 23.1\% in dental alveolar surgery. $24.2 \%$ wanted to work in salivary gland surgery, $23.1 \%$ in craniofacial surgery and 20.9\% in cleft surgery. $7.7 \%$ wanted to work in TMJ surgery and $6.6 \%$ in oral medicine. $20.9 \%$ were keen to be involved in academia (teaching) and 9.9\% in academia (research) (Fig. 2). $76.6 \%$ of trainees intended to complete a fellowship.

\section{Training background}

Trainees can initially qualify in medicine or dentistry. Figure 3 shows the year of graduation of trainees from their primary degree. The vogue has been dentistry first; however there is a sharp rise in the number graduating from dentistry in 2010 who are currently OMFS StRs.

Almost all trainees had obtained more than six months experience in oral and maxillofacial surgery at SHO level (Fig. 4).

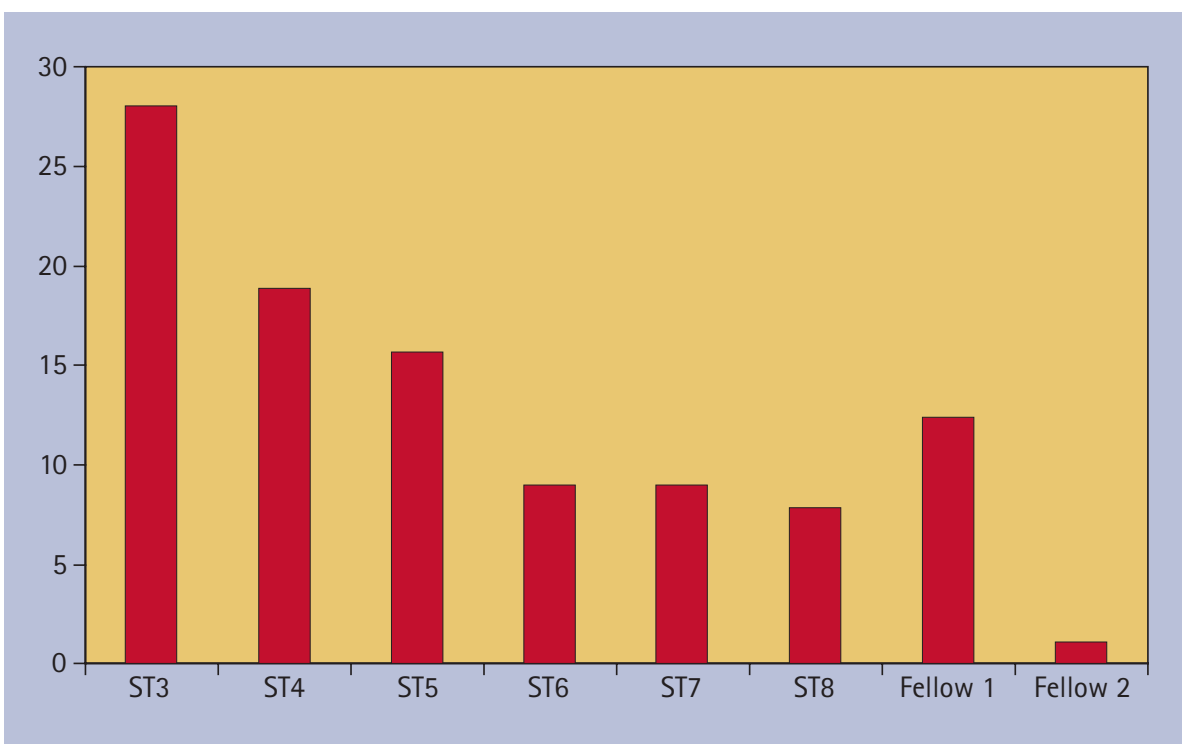

Fig. 1 The number of trainees in each year of specialist training

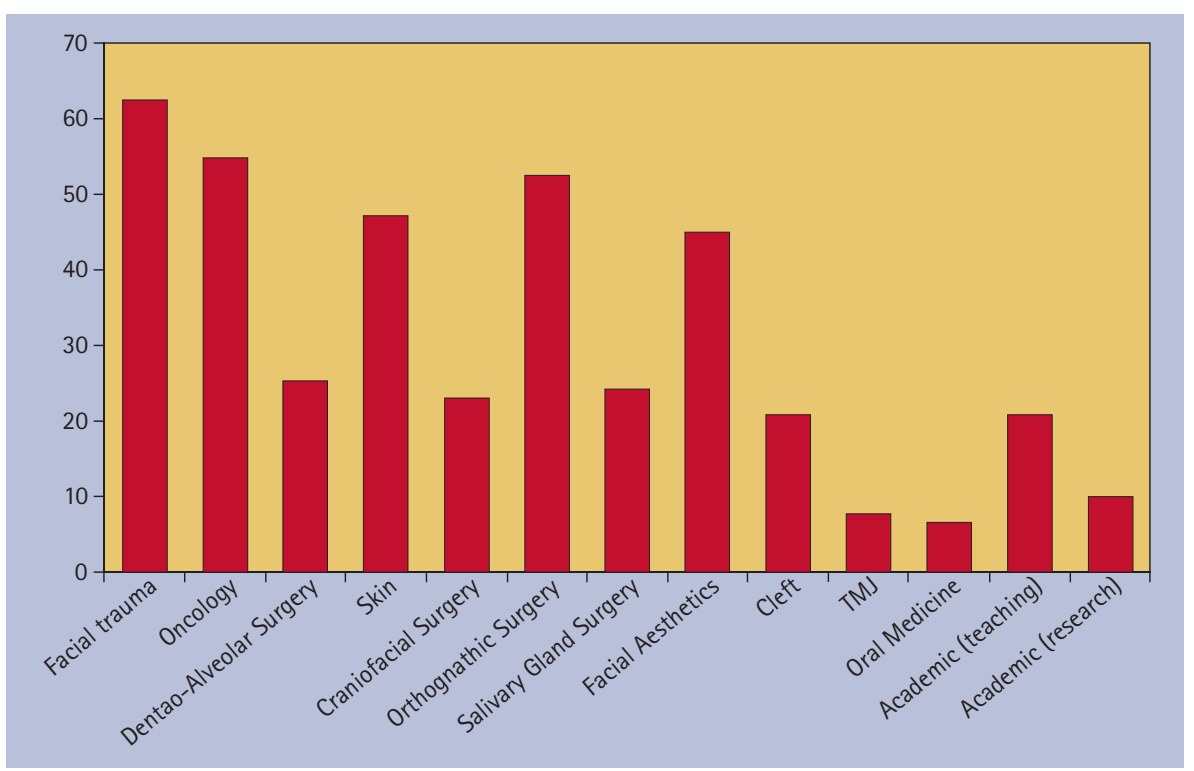

Fig. 2 Percentage of trainees keen to work in various areas of OMFS

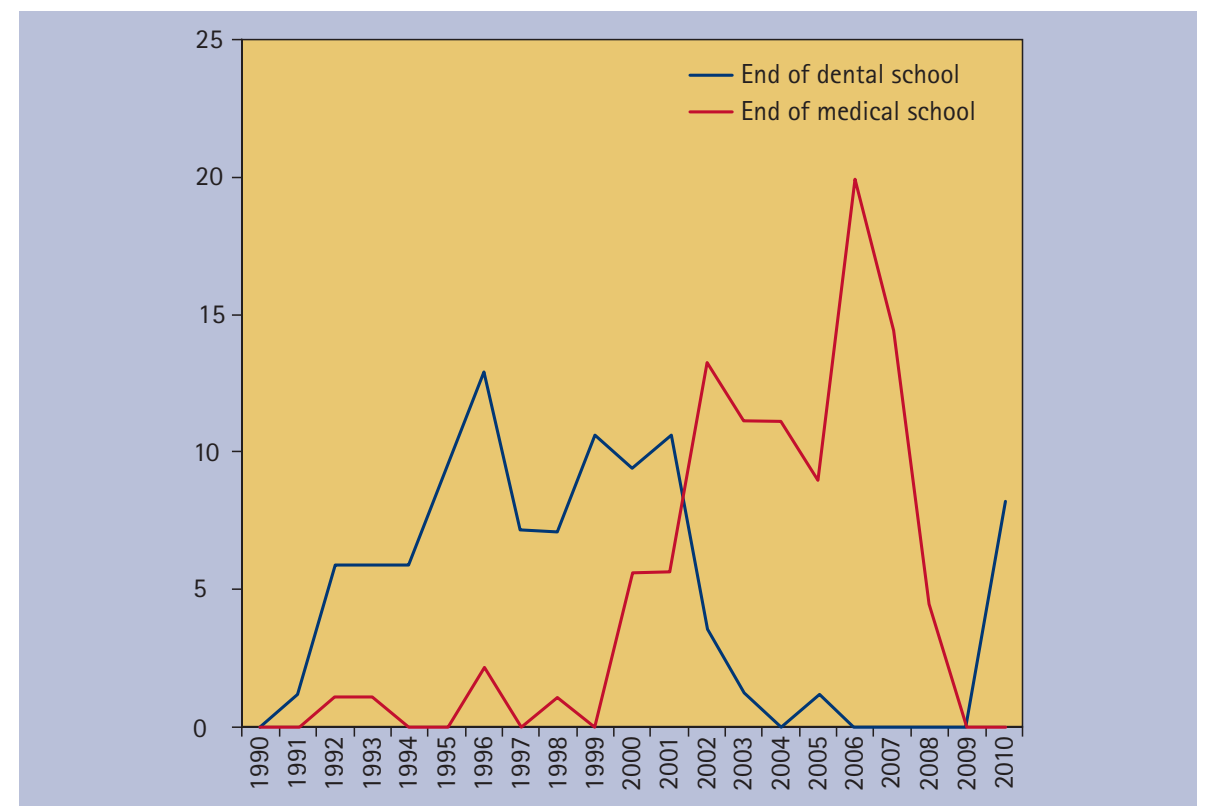

Fig. 3 Number of graduates of medical or dental school by year 


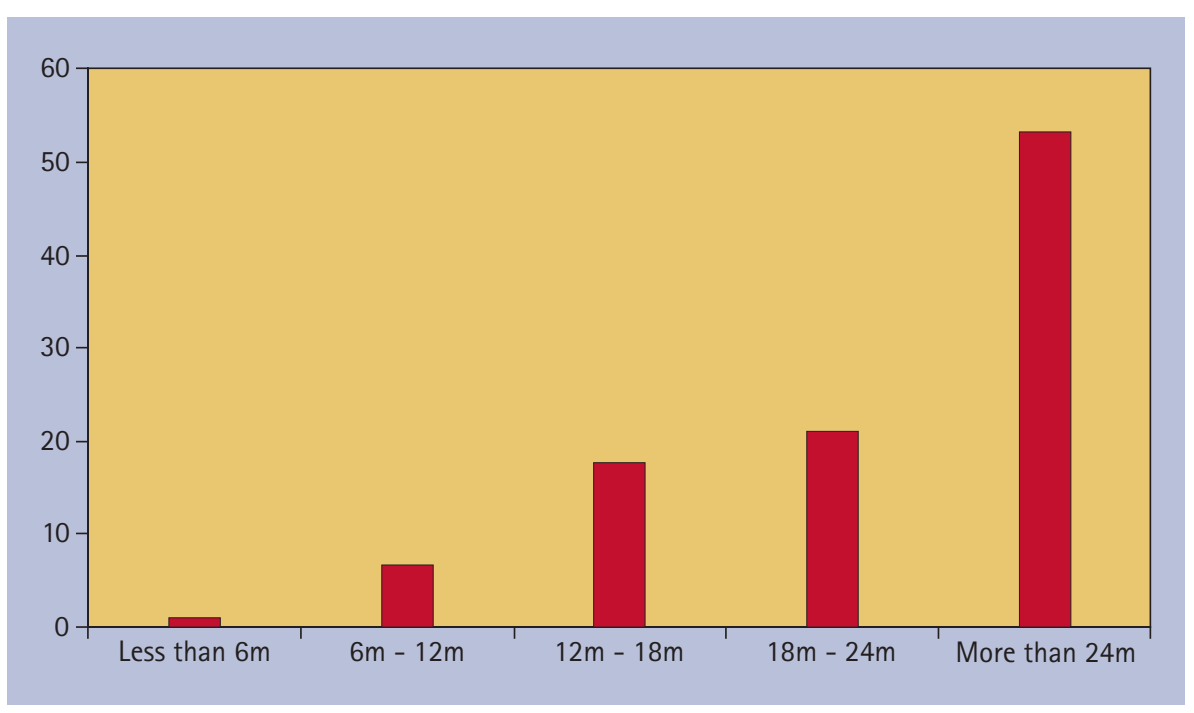

Fig. 4 Months of experience in OMFS at SHO Level

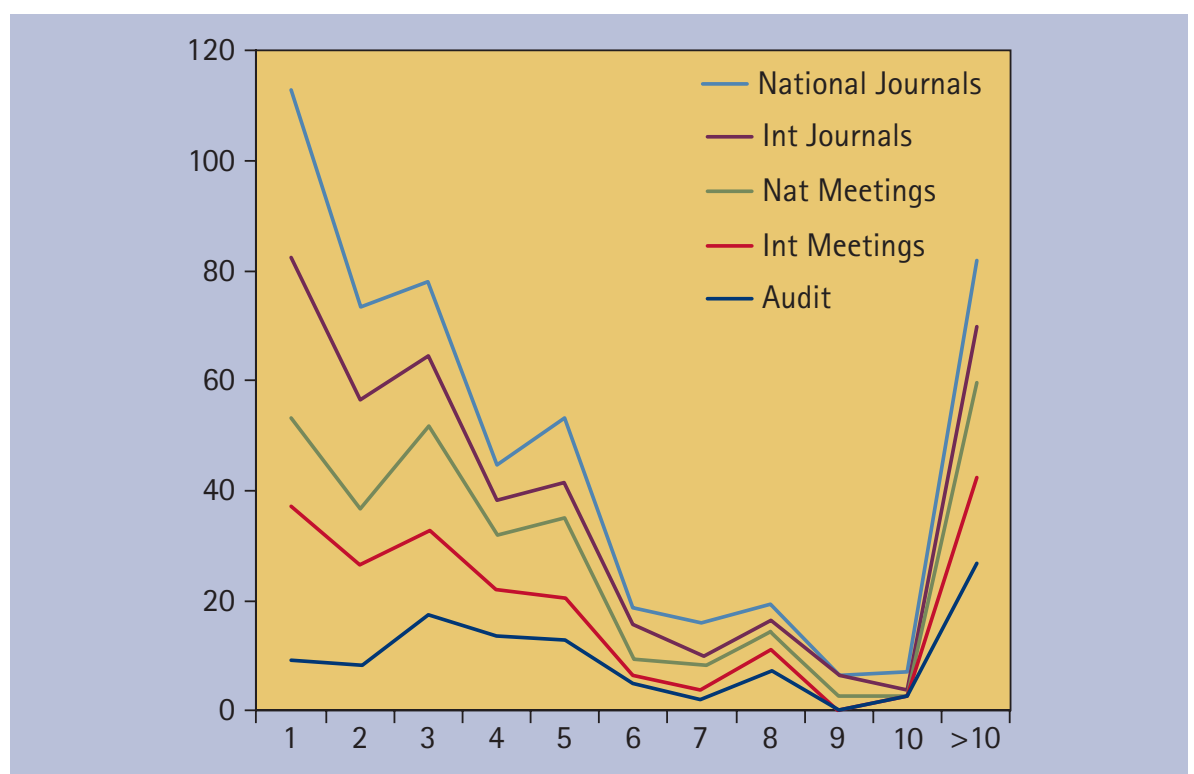

Fig. 5 Number of trainees and the number of publications, presentations and audits they have obtained

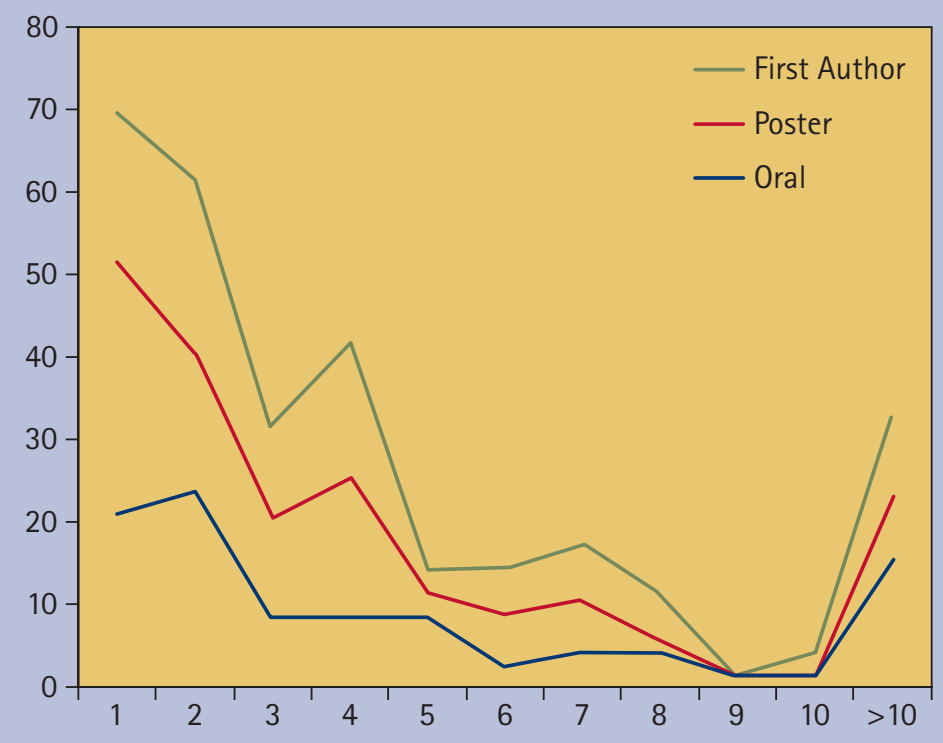

Fig. 6 Number of trainees and the number of first author publiciations, posters and oral presentations

\section{Qualifications}

In terms of additional degree qualifications $15.2 \%$ had obtained a BA or BSc and 8.5\% had a MSc or MA. 2.8\% had a MD and 4.7\% had a PhD.

In terms of memberships and fellowships offered by Royal colleges: 100\% had membership of the Royal College of Surgeons (MRCS) and 90.8\% had membership of the Faculty of Dental Surgeons (MFDS). 7.6\% had a diploma in otolaryngology - head and neck surgery (DOHNS) and 8.5\% were fellows of the Faculty of Dentistry, Royal College of Surgeons in Ireland (oral surgery with oral medicine) (FFDRCSI[OSOM]). 23.8\% were fellows of the Royal College of Surgeons (FRCS[OMFS]) which is the exit examination in OMFS and usually taken in the last few years of training. It is not possible to complete training without this exam.

\section{Academic background}

Figures 5 and 6 show the number of trainees who have achieved publications, presentations and audits. 9.3\% of trainees had taken time out of formal research. The range of time out of research was 3-60 months, with an average of 25.6 months. $22.1 \%$ of trainees had obtained grants, with one third of these obtaining over £50,000. 43.9\% were awarded less than $£ 5,000$ and $27.8 \%$ were awarded between $£ 5,000$ and $£ 10,000$.

In terms of prizes awarded, 72.1\% received them during their first degree and $50 \%$ during their second degree. Almost a third (32\%) received awards before and $21.4 \%$ since starting their specialist training programme.

\section{DISCUSSION}

Trainees in oral and maxillofacial surgery have a very high level of academic achievement. This may be a beneficial side effect of the large proportion of career time that is spent before obtaining a place on a specialist training programme. However, it has been noted that training in OMFS is no longer than other surgical specialties. ${ }^{3}$ Yet, Modernising Medical Careers has reduced the time spent in basic surgical training from an average of 30 months to 12 months. ${ }^{4}$ Indeed much of the curricula relating to postgraduate dental training will have been covered in part during the postgraduate foundation, general surgical, and oral and maxillofacial surgery training., 
Despite this, very few are keen to have an academic portion to their career. Indeed only $9.9 \%$ are keen for research to be a part of their consultant career. However, just over a fifth (20.9\%) are keen for teaching and education to be a formal part of their consultant career. Only 5.4\% of trainees in radiology reported a desire to be involved in academic radiology.

Sixteen percent of trainees have obtained a higher degree, with 7.5\% having obtained a MD or PhD. Given the length of undergraduate training required it is of no surprise that trainees may be reluctant to pursue research degrees which may further increase the amount of time in non-clinical training. As with other specialties there is a crisis in academic oral and maxillofacial surgery, and current academic training schemes in other surgical specialties could help. A more contemporary approach is needed in oral and maxillofacial surgery, and opportunities for intercalated or concurrent degrees by thesis alongside the second undergraduate degree must be sought with some urgency. An example of this is the concurrent BMedSci that is offered alongside the undergraduate medical programme at Nottingham University.

SHOs in plastic surgery and specialty trainees in radiology have a similar academic attainment in terms of higher degrees. ${ }^{1,2}$ Six percent of plastic trainees at SHO level, had obtained a higher degree whereas $19.2 \%$ of radiology trainees had obtained one.

Where oral and maxillofacial trainees are doing well is in obtaining research grants; $22.1 \%$ of trainees had received funding for such and 16.3\% of radiology trainees had done so.

Being involved in teaching the wide range of head and neck topics within the medical school curriculum would give medical trainees a clear image of what we do and what we do not do within OMFS.In the USA only 36\% of OMFS residency programmes have scheduled research time and this was usually three months or less. ${ }^{8}$ Residents felt that the scheduled research time was inadequate. Both residents and programme directors felt that research experience was useful for those considering an academic career, but less so for those considering a career in private practice. This study had a low response rate in both the programme directors (43\%) and residents (29\%).

Furthermore, a study looking at the characteristics of residents and residency programmes in the USA that led to graduating residents entering into academic positions ${ }^{9}$ identified that 1 in 12 graduates entered academic positions, with half of all residency programmes having no graduates entering academia. They identified that the number of faculty members who were dual qualified, had a $\mathrm{PhD}$ or held grants were not related to residents entering academic positions. They did identify that the number of faculty members involved in research was related to graduating residents entering academia.

It is clear that there are many reasons, some personal, that effect which trainees enter academic career pathways. Opportunities need to be available for those who self-select into research, education and teaching ${ }^{10}$ to foster their motivation and enthusiasm. Role models and mentors in surgery are useful ${ }^{11,12}$ and named individuals should be available at local department and hospital level, as well as at specialty and national level.

\section{CONCLUSION}

Future applicants to training programmes in oral and maxillofacial surgery in the United Kingdom are now aware of the level of competition. Furthermore, trainees in other specialties who may compete with oral and maxillofacial trainees for places on fellowships in head and neck oncology, cleft surgery and facial aesthetics will be aware of the strong academic background that exists in the specialty. This will assist in terms of manpower planning of oral and maxillofacial services nationally.
Those involved in recruitment into training programmes will also be able to see the level of competition.

Across the world, oral and maxillofacial surgery is not immune to the 'academic crisis' that exists in other surgical specialties. Completion of higher degrees and entry in to academic careers should be encouraged among trainees who show enthusiasm. Informal and formal research networks should be encouraged to expand. In addition the extended pre-specialist training period could be taken advantage of to consolidate and develop the evidence base for our patients.

1. McWeeney D M, Walker T W M, Gilbert F J, McCarthy P A. Radiology trainees in the UK and Ireland: academic background, publication rates and research plans. Br J Radio/ 2009; 82: 1033

2. Whitaker IS, Eyre J R, Izadi D, Rhodes N D, Foo I T. Plastic surgery senior house officers in the UK and Ireland: academic background, publication rates and research plans. Br J Plast Surg 2004; 57 : 139-142.

3. Cameron M, Westcott L. Maxillofacial training is no longer than other surgical specialties. Ann $R$ Coll Surg Eng/ 2008; 90: 146-149.

4. Dhanda J, Opie N, Webster K, Tanday A, Mumtaz S, Visram S. Impact of modernizing medical careers on basic surgical training and experience of oral and maxillofacial higher surgical trainee. Br J Oral Maxillofac Surg 2011; 49: 62-64.

5. Varley I S, Argiris K, Walker T W, Magennis P. Qualitative Comparison of Curricula in oral and maxillofacial surgery. Part 1: dental foundation training. Br J Oral Maxillofac Surg 2012; 50: 464-467.

6. Walker T W, Varley I S, Argiris K, Magennis P. Qualitative Comparison of curricula in oral and maxillofacial surgery training. Part 2: Oral Surgery. Br J Oral Maxillofac Surg 2012; 50: 468-469.

7. Shanks L A, Walker T W, McCann P J, Kerin M J. Oral cavity examination: beyond the core curriculum? $\mathrm{Br}$ J Oral Maxillofac Surg 2011; 49: 640-642.

8. Mohammad A E, Best A M, Laskin D M. Attitudes and Opinions of Residency Directors and Residents about the importance of research in oral and maxillofacial surgery residencies. J Oral Maxillofac Surg 2011; 69: 2064-2069.

9. Sarraf A A, Abubaker A O, Laskin D M, Best A. Characteristics of Oral \& Maxillofacial Surgery Residencies That Results in Graduating Residents Entering Academic Positions. J Oral Maxillofac Surg 2011; 69: 2271-2274

10. Walker T W, Cascarini L, Brennan P A. Educational Paper: Research in Oral \& Maxillofacial Surgery. Br J Oral Maxillofac Surg 2010; 48: 629-632.

11. Healy N A, Glynn R W, Malone C, Cantillon P, Kerin $M$ J. Surgical Mentors and Role models: prevalence, importance and associated traits. J Surg Educ 2012; 69: 633-637.

12. Healy N A, Cantillon P, Malone C, Kerin M J. Role models and mentors in surgery. Am J Surg 2012; 204: 256-261. 